\title{
CORPO DOCENTE DE 1911
}

DIRECTOR

Dr. Antonio Dino da Costa Bueno

LENTES CATHEDRATICOS

Dr. Brasilio Augusto Machado d'Oliveira

Dr. Joâo Mendes de Almeida Junior

Dr. José Luiz de Almeida Nogueira

Dr. Uladisláu Herculano de Freitas

Dr. Antonio Amancio Pereira de Carvalho

Dr. Antonio Januario Pinto Ferraz

Dr. Manoel Pedro Villaboim

Dr. José Machado de Oliveıra, em disponibilidade

Dr. Ernesto Moura, em disponibilidade

Dr. Joāo Pedro da Veiga Filho, em disponibilidade

Dr. Aliredo de Barros de Oliveira Lima

Dr. José Ulpiano Pinto de Souza

Dr. Candido Nazianzeno Nogueira da Motta

Dr. José Mariano Corrêa de Camargo Aranha

Dr. Gabriel José Kodrigues de Rezende

Dr. Reynaldo Porchat

Dr. José Bonifacio de Oliveira Coutinho

Dr. João Braz de Oliveira Arruda

Dr. Luiz Barboza da Gama Cerqueira

Dr. Estevam de Araujo Almeida

Dr. José Mendes.

LENTES SOBSTITUTOS

Dr. Raphael Corrêa da Silva

Dr. José de Alcantara Machado d'Oliveira

Dr Dario Sebastiāo de ()liveira Ribeiro

Dr. Frederico Vergueiro Steidel

Dr. Raphael Corrêa de Sampaio

Dr. Manoel Pacheco Prates.

SECRETARIO

Bacharel Julio Joaquim Gonçalves Maia.

SUB-SECRETARIO

Bacharel Aureliano Amaral.

BIBLIOTHECARIO

Bacharel Eugenio Manoel de Toledo.

SUB-BLBLYOTHECARYO

Bacharel Luiz de Andrade Vasconcellos Junior.

THESOOREIRO

Bacharel Honorio de Castilhos. 